\title{
Crianças em risco psicossocial associado à violência doméstica: o desempenho escolar e o autoconceito como condições de proteção
}

\author{
Rute Grossi Milani \\ Centro Universitário de Maringá \\ Sonia Regina Loureiro \\ Universidade de São Paulo
}

\begin{abstract}
Resumo
Objetivou-se avaliar crianças com história de risco psicossocial, associado à violência doméstica, que implicou em notificação ao Conselho Tutelar, e compará-las com um grupo de crianças sem história de risco psicossocial, quanto ao autoconceito e ao desempenho escolar. Foram avaliadas 40 crianças, de ambos os sexos, de oito a doze anos, que residiam com pelo menos um dos pais biológicos, distribuídas em dois grupos, um com histórico de violência doméstica; e outro, sem história de risco psicossocial relatada. Procedeu-se à aplicação da Escala Piers-Harris de Autoconceito e do Teste de Desempenho Escolar. As crianças com história de violência doméstica apresentaram um autoconceito mais negativo na área comportamento e mais dificuldade no desempenho escolar na área de escrita. Tais dificuldades sugerem prejuízos em áreas que deveriam funcionar como proteção, ou seja, que essas crianças não contam com alguns recursos essenciais para enfrentarem as tarefas desenvolvimentais da idade escolar.
\end{abstract}

Palavras-chave: violência doméstica; autoconceito; desempenho escolar.

\begin{abstract}
Children in psychosocial risk associated with domestic violence: school achievement and self-concept as protection conditions. The goal was to assess children with psychosocial risk history, associated with domestic violence, which implied in notifying the Child Protection Agency, and to compare it to a group of children without a history of psychosocial risk, as for the self-concept and school achievement. Forty children were assessed, both girls and boys, from eight to twelve years of age, who lived with at least one biological parent, distributed in two groups: one with a history of domestic violence and the other one without a reported psychosocial risk. Two tests were performed: The Piers-Harris Children's Self-concept Scale and the School Performance Test. The children with a history of domestic violence presented a more negative self-concept in behavior and more difficulty in school achievement in writing. Such difficulties suggest impairments in areas which should work as protection, i.e., these children do not count with some of the essential resources to face developmental tasks at school age.
\end{abstract}

Keywords: domestic violence; self-concept; school achievement.

$\mathrm{N}$ cotidiano, muitas crianças vivenciam eventos estressores, dentre eles, destaca-se a violência no contexto familiar. Ela pode envolver atitudes de abuso ou negligência dos responsáveis direcionados à criança, bem como a violência interparental presenciada pela criança.

De acordo com Deslandes (1994), podem-se considerar quatro tipos de violência doméstica direcionados à criança: violência física, violência psicológica, negligência e violência sexual. Apesar das tentativas de classificar a violência doméstica, observam-se muitas controvérsias que têm influenciado a operacionalização da experiência de maus-tratos, reconhecendose que é um fenômeno heterogêneo e complexo (Cicchetti \& Rizley, 1981). Milani e Loureiro (2008), ao analisarem os tipos de violência vivenciados pelas crianças atendidas pelo Conselho Tutelar, observaram a atuação paralela de diferentes formas de violência, como a violência doméstica direcionada à criança e a violência interparental. No referido estudo, as autoras verificaram, ainda, as condições familiares das crianças atendidas por violência doméstica, e observaram que esta forma de violência pode estar relacionada a múltiplas adversidades como o conflito parental recorrente e o uso abusivo de álcool e drogas, mostrando que, ao se referir à violência doméstica como uma adversidade, quase sempre se faz presente um conjunto de condições negativas. Outros estudos têm destacado a complexidade das condições envolvidas no estudo da violência doméstica, apontando para a associação com fatores tais como a desigualdade social, problemas quanto à qualidade de vida e o comprometimento das relações intrafamiliares (Roque \& Ferriani, 2002).

Muitos estudos vêm sendo desenvolvidos com a finalidade 
específica de delimitar as conseqüências da violência doméstica sobre o desenvolvimento infantil. McCloskey, Figueiredo e Koss (1995) constataram a influência direta da violência doméstica na saúde mental das crianças e relataram a importância de se considerar a violência interparental presenciada pela criança, chamando a atenção para o fato que as crianças de lares violentos estão sob risco elevado de sofrerem violência de ambos os pais, principalmente a violência física.

Szyndrowski (1999), ao descrever características comportamentais observadas em crianças que testemunham violência doméstica, destaca as seguintes manifestações: agressividade, medo intenso, baixa auto-estima, desconfiança nas relações íntimas, medo do futuro, reclamações psicossomáticas e problemas relativos à autoridade.

A associação entre exposição à violência interparental e problemas acadêmicos em crianças foi estudada por Kernic et al. (2002). As crianças expostas à violência apresentaram maior absenteísmo e maior probabilidade de serem suspensas da escola devido a problemas de comportamento. Entretanto, não foram encontradas dificuldades em relação à performance acadêmica.

Brancalhone, Fogo e Williams (2004) avaliaram o desempenho acadêmico de crianças expostas à violência conjugal. Os autores não encontraram diferenças no desempenho acadêmico delas quando comparadas a outras de famílias não violentas, porém, detectaram diferenças entre os grupos quanto à avaliação do professor. Os autores hipotetizam que conhecimentos prévios sobre a história de vida das crianças possam ter influenciado a avaliação do professor, bem como a influência de outros fatores subjetivos, como o nível de exigência do professor e sentimentos despertados em relação aos alunos. Comentam que tais sentimentos podem contribuir para que os professores tenham baixas expectativas em relação às crianças expostas à violência conjugal.

Salzinger, Feldman, Ng-Mak, Mojica e Stockhammer (2001) avaliaram a expectativa social e o status social de crianças vítimas de violência doméstica no grupo. Crianças abusadas fisicamente apresentaram-se menos propensas a esperar que seus colegas de classe as escolhessem positivamente; revelaram, ainda, menor tendência a se engajar em comportamento pró-social.

O comportamento da criança no contexto escolar e, mais especificamente, o seu ajustamento acadêmico foi alvo de atenção de Shonk e Cicchetti (2001), que constataram prejuízos nesse ajustamento. As crianças maltratadas apresentaram menos engajamento que o grupo de comparação, ou seja, menos motivação para se envolver de forma autônoma com comportamentos que levam ao aprendizado.

Ao analisar estes estudos, observam-se associações significativas entre violência doméstica direcionada à criança, autopercepções relativas ao relacionamento social e prejuízos no ajustamento escolar, porém não foram encontradas dificuldades quanto ao desempenho escolar.

O estudo sobre os riscos psicossociais, dentre eles, a violência doméstica, constitui-se área de interesse da psicopatologia do desenvolvimento, especialmente no que se refere à exploração das associações entre a violência, o desempenho escolar e o autoconceito, dada a relevância destas variáveis como indicadores da realização das tarefas de desenvolvimento próprias da fase escolar.

Dentre tais tarefas, a adaptação à vida escolar é considerada socialmente decisiva para o desenvolvimento das crianças, no período dos seis aos 12 anos. Erikson (1976), apoiado em um enfoque psicossocial do desenvolvimento, considera que a personalidade se desenvolve em etapas caracterizadas por crises e que nos anos iniciais de escolarização fundamental predomina a vivência de conflitos entre o senso de realização e o senso de inferioridade, expressando-se pelo desejo das crianças de mostrarem-se competentes e com capacidade produtiva. Tratase de um período em que a criança busca o reconhecimento social através de sua capacidade de se preparar para o trabalho no mundo adulto. Neste sentido, o desempenho acadêmico, o ajustamento ao ambiente escolar e a capacidade de estabelecer relações sociais gratificantes são importantes indicadores dos recursos de adaptação (Jacob \& Loureiro, 1996).

As dificuldades de adaptação às demandas dessa fase evidenciam comprometimentos no desenvolvimento da criança, podendo implicar problemas de aprendizagem e/ ou de comportamento. $\mathrm{Na}$ fase escolar, as dificuldades de desempenho e de comportamento se configuram como condições de vulnerabilidade ao desenvolvimento, dada a relevância da tarefa de produtividade para este momento da vida. Para o enfrentamento destas demandas, as crianças contam com recursos pessoais e ambientais.

Dentre os fatores pessoais que têm sido destacados como variáveis importantes para a avaliação dos recursos internos da criança situa-se o autoconceito (Candieux, 1996; Jacob, 2001). Ao longo do desenvolvimento infantil, a criança experimenta uma variedade de relações interpessoais, na família e na escola, que se configuram como o contexto de modulação do autoconceito. Chapman (1988) e Oliveira (2000) descrevem o constructo autoconceito como um conjunto de crenças e autopercepções construídas ao longo da vida, envolvendo atitudes, sentimentos e conhecimentos sobre capacidade, habilidades e aceitação social, que direcionam a conduta dos indivíduos, permitindo que assumam novas responsabilidades na vida.

De uma maneira muito simplificada, pode-se dizer que o autoconceito reflete a avaliação cognitiva que cada indivíduo faz de seus atributos, habilidades e características frente a domínios específicos, como a aparência física, a competência acadêmica ou para o esporte, enquanto a auto-estima parece refletir o significado afetivo mais geral que estes atributos, habilidades e características têm para o indivíduo. Campbell e Lavallee (1993), e Harter (1993) abordam estes dois construtos como multidimensionais, apresentando-os como correlacionados positivamente com relação causal recíproca e sistemática. Apontam, com base em teóricos da personalidade, que uma visão positiva de si ou uma boa identidade tem íntima relação com uma auto-estima positiva.

Ressalta-se, porém, que a avaliação positiva do autoconceito pela criança, em discrepância com as avaliações externas, pode refletir imaturidade cognitiva para perceber seu real desempenho, ou, ainda, este autoconceito positivo pode estar desempenhando uma função protetora, devido à dificuldade de reconhecer suas falhas acadêmicas e sociais (Gresham, Lane, Mac Millan, 
Bocian, \& Ward, 2000). Neste sentido, o comportamento pouco adaptado e com características anti-sociais pode se mostrar associado à popularidade da criança. Então, o que seria um suposto problema de ajustamento para os pais e professores, pode não ser assim considerado pela criança e por seus pares (Rodkin, Farmer, Pearl, \& Arcker, 2000; Stevenato, Loureiro, Linhares, \& Marturano, 2003). Por essa razão, a relação entre autoconceito e ajustamento é mais bem explicada pela consistência ou discrepância entre as auto-avaliações e as avaliações externas (Gresham et al., 2000).

Considera-se que a adaptação da criança aos eventos de vida estressantes, como a violência doméstica, e a forma como se coloca frente às situações de seu cotidiano, são influenciadas pela avaliação cognitiva que ela faz de seus atributos e habilidades, assim, as estratégias de enfrentamento empregadas estão diretamente ligadas ao seu autoconceito. Nesse sentido, os recursos pessoais das crianças favorecem respostas positivas às situações de adversidade, que podem minimizar o impacto do estressor.

Neste contexto, o presente estudo teve como objetivo avaliar um grupo de crianças com história de risco psicossocial, associado à violência doméstica, que implicou em medidas legais junto ao Conselho Tutelar, e compará-lo quanto às autopercepções relativas ao autoconceito e às capacidades fundamentais para o desempenho escolar, com um grupo de crianças sem história de risco psicossocial relatada.

\section{Método}

Trata-se de um estudo prospectivo, de comparação entre grupos. O estudo foi aprovado pelo Comitê de Ética em Pesquisa da Universidade Estadual de Maringá.

\section{Participantes}

Foram avaliadas 40 crianças, de ambos os sexos, na faixa etária de oito a 12 anos incompletos, distribuídas em dois grupos: 20 incluídas no Grupo $1(\mathrm{G} 1)$ = risco psicossocial -violência doméstica, atendidas junto ao Conselho Tutelar do Município de Maringá - Paraná; e 20 incluídas no Grupo 2 (G2) = sem história de risco psicossocial relatada. Procurou-se, na constituição do grupo de comparação (G2), pareá-lo quanto ao sexo e balanceálo quanto à idade atual, à série escolar, ao nível cognitivo, à residência com pelo menos um dos pais biológicos, à ausência de deficiência física ou mental evidente e a características socioeconômicas familiares semelhantes.

Para a seleção dos 40 participantes, foram contatadas 106 crianças, 58 como possíveis integrantes do G1 e 48 como possíveis integrantes do G2. Para G1 e G2 foram excluídas do estudo crianças institucionalizadas, com história de adoção, que sofreram violência sexual, com deficiência física ou mental evidente, e como condição de inclusão geral para G1 e G2 a residência com pelo menos um dos pais biológicos.

Foram incluídos em cada grupo 12 meninos e oito meninas. A idade das crianças, no momento da coleta de dados, variou de oito anos e seis meses a 11 anos e oito meses, observando-se, nos dois grupos, distribuição semelhante. As crianças apresentavam entre cinco e nove anos quando ocorreu a denúncia de violência doméstica ao Conselho Tutelar. Quanto à escolaridade das crianças, predominou a terceira e a quarta série e, também, não se observou diferença relevante entre os grupos.

Quanto à avaliação intelectual, os dados também foram semelhantes para as crianças dos dois grupos. Em ambos, a pontuação média foi bastante próxima, correspondendo ao nível médio e ao percentil 50 .

Os critérios de composição dos grupos foram analisados de forma sistemática, ou seja, foi realizado tratamento estatístico dos dados relativos à idade e ao nível intelectual da criança, à escolaridade da criança e dos pais, e ao nível socioeconômico da família. Não foram observadas diferenças estatisticamente significativas em relação a tais variáveis analisadas.

\section{Instrumentos}

Para a seleção dos participantes, foram utilizados os seguintes instrumentos:

Com as crianças do G1 e do G2. Matrizes Progressivas Coloridas de Raven - Escala Especial. Este instrumento foi adaptado para avaliação do nível intelectual de crianças brasileiras (Angelini, Alves, Custódio, Duarte, \& Duarte, 1999). É composto por 36 itens, divididos em três séries.

\section{Para a coleta de dados propriamente dita}

Com as crianças.

Escala Infantil Piers-Harris de Autoconceito. - "O que eu sinto sobre mim mesmo" - (Piers, 1984), traduzida e adaptada para o nosso meio por Jacob e Loureiro (1999). Inclui 80 itens, divididos em agrupamentos relativos a seis categorias de Autoconceito: comportamento; status intelectual e acadêmico; aparência física e atributos; ansiedade; popularidade; felicidade e satisfação. Em cada item, é solicitado que a criança responda "sim" ou "não", de acordo com a maneira como se sente em relação a si, na maioria das situações.

Teste de Desempenho Escolar (TDE) - (Stein, 1994). É composto de três subtestes: escrita: escrita do próprio nome e de palavras isoladas, apresentadas sob a forma de ditado; aritmética: solução oral de problemas e cálculo de operações aritméticas por escrito; leitura: reconhecimento de palavras isoladas do contexto. Os itens dos subtestes estão organizados em ordem crescente de dificuldade e são apresentados à criança, independente de sua série.

\section{Procedimento de coleta de dados}

As crianças foram identificadas através do banco de dados do Conselho Tutelar de Maringá, mediante autorização do Juiz da Vara da Infância e Juventude de Maringá.

As atividades de coleta de dados foram distribuídas da seguinte forma:

1. a sessão: A seleção das crianças foi feita através da aplicação do teste Matrizes Progressivas. A aplicação foi realizada conforme as recomendações técnicas e os resultados foram comparados às normas brasileiras, propostas por Angelini et al. (1999). Nessa sessão, foi realizado um breve rapport, obtendo-se os dados de identificação e buscando-se o estabelecimento de um contato produtivo com a criança. 
2. ${ }^{\text {a }}$ sessão: Os itens da Escala Infantil Piers-Harris de Autoconceito foram apresentados através de fita gravada na voz da avaliadora, evitando, assim, variações de leitura quanto a entonações e pausas. A criança pôde ouvir os itens repetidas vezes, caso necessário. A avaliadora anotou as respostas no protocolo.

Foi feita, também, a aplicação do Teste de Desempenho Escolar, apresentado através de um caderno contendo os três subtestes. Iniciou-se sempre pelo primeiro item de cada escala. Encerrava-se a avaliação de acordo com o nível que o examinando conseguia atingir.

\section{Procedimento de análise de dados}

Os instrumentos padronizados foram codificados e quantificados de acordo com as recomendações de cada técnica.

Foi verificada a normalidade dos dados através do teste de Kolmogorov-Smirnov (KS). O teste de KS evidenciou predominantemente uma distribuição normal dos dados, sendo recomendado o emprego do teste $t$ de student para amostras independentes. Os dados que não apresentaram uma distribuição normal foram analisados por meio do Teste Não-Paramétrico $U$ de Mann-Whitney. Em todos os procedimentos estatísticos adotou-se o nível de significância de $p \leq 0,05$.

\section{Resultados}

Serão apresentados separadamente os dados de comparação dos grupos, G1 = risco psicossocial - violência doméstica e G2 $=$ sem história de risco psicossocial relatada, relativos a cada instrumento: Escala Infantil Piers-Harris de Autoconceito e Teste de Desempenho Escolar.

\section{Autoconceito}

\section{Escala Infantil Piers-Harris de Autoconceito}

A comparação dos grupos quanto ao escore total e aos escores parciais das categorias de Autoconceito é apresentada na tabela 1.

Não foi observada diferença estatisticamente significativa na comparação dos grupos em relação ao escore total do

Tabela 1

Autoconceito: Média, Desvio Padrão e Mediana relativos ao Escore Total, às seis categorias de Autoconceito e às comparações dos grupos G1 e G2.

\begin{tabular}{|c|c|c|c|c|c|c|c|c|}
\hline \multirow[t]{2}{*}{ Áreas } & \multicolumn{3}{|c|}{ G1 } & \multicolumn{3}{|c|}{$\mathrm{G} 2$} & \multirow[t]{2}{*}{$p$} & \multirow[t]{2}{*}{ Comparações } \\
\hline & Média & $(D P)$ & Mediana & Média & $(D P)$ & Mediana & & \\
\hline Total & 54,60 & 13,32 & 56,5 & 60,70 & 8,09 & 59,5 & 0,09 & NS \\
\hline Comportamento & 11,35 & 3,43 & 11,5 & 13,60 & 2,16 & 14 & 0,02 & $\mathrm{G} 1<\mathrm{G} 2 *$ \\
\hline Status Intelectual & 12,20 & 3,80 & 13 & 13,70 & 2,25 & 13,5 & 0,14 & NS \\
\hline Aparência & 9,40 & 2,92 & 10 & 8,25 & 2,82 & 9 & 0,21 & NS \\
\hline Ansiedade & 7,95 & 2,32 & 8 & 9,10 & 2,15 & 9 & 0,11 & NS \\
\hline Popularidade & 7,65 & 2,20 & 7 & 7,70 & 2,00 & 7 & 0,94 & NS \\
\hline $\begin{array}{l}\text { Felicidade e } \\
\text { Satisfacão•• }\end{array}$ & 8,20 & 1,96 & 9 & 8,65 & 1,60 & 9 & 0,42 & NS \\
\hline
\end{tabular}

Teste $t$ de student $\quad \mathrm{NS}=$ não significativo $\quad * p \leq 0,05$

- Teste Não-Paramétrico $U$ de Mann-Whitney

$\mathrm{G} 1=$ risco psicossocial - violência doméstica

$\mathrm{G} 2=$ sem história de risco psicossocial relatada

autoconceito, indicando semelhança quanto à avaliação global de si.

Observou-se diferença estatisticamente significativa entre os grupos apenas em relação à categoria "comportamento". As crianças do G1 apresentaram um autoconceito relativo ao comportamento mais negativo em comparação às crianças do G2, indicativo de mais dificuldade nessa área. Nas demais categorias, não foram observadas diferenças estatisticamente significativas quando da comparação dos grupos.

Os grupos foram comparados quanto a cada um dos itens da escala. Estão apresentados na tabela 2 os itens com diferenças significativas.

Dentre os 80 itens que compõem a escala, na comparação dos grupos, 11 apresentaram diferença estatisticamente significativa.
Considerando as áreas, observou-se uma avaliação mais negativa do G1 em relação ao G2 na auto-avaliação relativa aos itens das áreas "comportamento" e "status intelectual e acadêmico". O G1 apresentou uma avaliação mais positiva em três itens, que se incluem na área "aparência Física e atributos", indicando um autoconceito mais positivo quanto a esta área na comparação com as crianças do G2.

\section{Desempenho Escolar}

Teste de Desempenho Escolar

Os dados relativos ao Teste de Desempenho Escolar são apresentados na tabela 3 .

Não foram observadas diferenças estatisticamente significativas na comparação dos grupos em relação ao escore total e nas áreas "aritmética" e "leitura. Observou-se diferença 
Tabela 2

Autoconceito: Proporção (Pr) de participantes do G1 e do G2 que pontuaram os itens com diferenças estatisticamente significativas

\begin{tabular}{|c|c|c|c|c|}
\hline $\begin{array}{c}\text { Área (Número de itens) } \\
\text { Itens• }\end{array}$ & $\begin{array}{c}\mathrm{G} 1 \\
\mathrm{Pr}\end{array}$ & $\begin{array}{l}\mathrm{G} 2 \\
\mathrm{Pr}\end{array}$ & $p$ & Comparações \\
\hline \multicolumn{5}{|l|}{ Comportamento (16 itens) } \\
\hline 12. Eu me comporto bem na escola (Sim) & 0,75 & 100 & 0,05 & $\mathrm{G} 1<\mathrm{G} 2 *$ \\
\hline 13. Geralmente a culpa é minha quando algo dá errado (Não) & 0,55 & 0,85 & 0,04 & $\mathrm{G} 1<\mathrm{G} 2 *$ \\
\hline 59. Minha família está triste comigo (Não) & 0,70 & 100 & 0,02 & $\mathrm{G} 1<\mathrm{G} 2 *$ \\
\hline \multicolumn{5}{|l|}{ Status Intelectual e acadêmico (17 itens) } \\
\hline 07. Eu fico nervoso quando a professora me chama (Não) & 0,55 & 0,90 & 0,01 & $\mathrm{G} 1<\mathrm{G} 2 *$ \\
\hline 12. Eu me comporto bem na escola ( $\mathrm{Sim})$ & 0,75 & 100 & 0,05 & $\mathrm{G} 1<\mathrm{G} 2 *$ \\
\hline 31. Na escola, eu fico no mundo da lua (Não) & 0,50 & 0,80 & 0,05 & $\mathrm{G} 1<\mathrm{G} 2 *$ \\
\hline 70. Eu leio bem $(\mathrm{Sim})$ & 0,50 & 0,85 & 0,02 & $\mathrm{G} 1<\mathrm{G} 2 *$ \\
\hline \multicolumn{5}{|l|}{ Aparência física e atributos (13 itens) } \\
\hline 15. Eu sou forte (Sim) & 0,85 & 0,55 & 0,04 & $\mathrm{G} 1>\mathrm{G} 2 *$ \\
\hline 57. Eu sou popular com os meninos ( $\mathrm{Sim})$ & 0,55 & 0,20 & 0,02 & $\mathrm{G} 1>\mathrm{G} 2 *$ \\
\hline 63. Eu sou um líder em brincadeiras e esportes (Sim) & 0,60 & 0,25 & 0,03 & $\mathrm{G} 1>\mathrm{G} 2 *$ \\
\hline \multicolumn{5}{|l|}{ Não incluídos nas categorias (15 itens) } \\
\hline 18. Eu sempre quero do meu jeito (Não) & 0,20 & 0,65 & 0,01 & $\mathrm{G} 1<\mathrm{G} 2 *$ \\
\hline 32. Eu atormento meu (s) irmão (s) e irmã (s) (Não) & 0,45 & 0,95 & 0,01 & $\mathrm{G} 1<\mathrm{G} 2 *$ \\
\hline
\end{tabular}

- Alguns itens repetiram-se por se enquadrar em mais de uma área

Teste Exato de Fisher; Teste $X^{2} \quad * p \leq 0,05$

$\mathrm{G} 1=$ risco psicossocial - violência doméstica

$\mathrm{G} 2=$ sem história de risco psicossocial relatada

( ) Resposta pontuada com valoração positiva

Tabela 3

Desempenho Escolar: Freqüencia (F) e Proporção (Pr) de sujeitos do G1 e do G2 com escores que atingiram o critério esperado para a escolaridade

\begin{tabular}{|c|c|c|c|c|c|c|}
\hline \multirow[t]{2}{*}{ Desempenho Escolar } & \multicolumn{2}{|c|}{ G1 } & \multicolumn{2}{|c|}{ G2 } & \multirow[t]{2}{*}{$p$} & \multirow[t]{2}{*}{ Comparações } \\
\hline & $\mathrm{F}$ & $\operatorname{Pr}$ & $\mathrm{F}$ & $\operatorname{Pr}$ & & \\
\hline Total & 7 & 0,35 & 12 & 0,60 & 0,11 & NS \\
\hline Escrita & 4 & 0,20 & 11 & 0,55 & 0,02 & $\mathrm{G} 1<\mathrm{G} 2 *$ \\
\hline Aritmética & 14 & 0,70 & 12 & 0,60 & 0,51 & NS \\
\hline Leitura & 9 & 0,45 & 12 & 0,60 & 0,34 & NS \\
\hline
\end{tabular}

estatisticamente significativa em relação à área "escrita". As crianças do G1 apresentaram valores inferiores às do G2, quando da comparação com os valores normativos.

\section{Discussão}

Constatou-se que a maneira como as crianças vítimas de violência doméstica estão lidando com as exigências próprias da idade escolar sugere dificuldades na capacidade adaptativa.

Analisando-se os dados decorrentes da Escala de Piers Harris de Autoconceito, observou-se que os dois grupos não se diferenciaram em relação ao escore total. Quanto às áreas específicas, observou-se diferença apenas na área relativa ao comportamento, as crianças vítimas de violência doméstica apresentaram uma avaliação menos positiva de si, julgandose culpadas pelos problemas que acontecem no cotidiano, avaliando negativamente seu comportamento na escola e, ainda, consideraram que a família está triste com elas.

Tais dados, relativos ao autoconceito, sugerem uma tendência das crianças vítimas de violência doméstica a atribuírem causas internas aos eventos percebidos como negativos, ou seja, consideram que a causa do evento está associada a algo interno, de sua responsabilidade. A tendência a fazer atribuições internas para resultados negativos, associada a uma visão negativa de si mesma, segundo Oliveira (2000), pode afetar as respostas eficazes diante das adversidades e pode 
implicar em uma diminuição na motivação, dificultando que assumam novas responsabilidades na vida.

As crianças vítimas de violência doméstica apresentaram, ainda, dificuldades relativas ao controle sobre os impulsos, expressas nos itens "querer sempre do seu jeito" e "atormentar o irmão". Essas afirmativas sugerem dificuldades quanto à socialização.

$\mathrm{Na}$ avaliação do autoconceito relativo à área escolar, as crianças vítimas de violência doméstica referiram mais dificuldades acadêmicas, tais como: ficar nervosa quando o professor chama, distração e julgar que lêem mal.

O autoconceito, a auto-estima positiva, a auto-eficácia, os recursos cognitivos e as habilidades de coping constituem fatores individuais de proteção e têm potencial para agir como moderadores dos efeitos negativos do stress (Masten, 2001). Na avaliação do autoconceito, as dificuldades mais significativas foram nas áreas comportamento e status intelectual. Tal dado sugere que as crianças vítimas de violência doméstica estão prejudicadas quanto aos recursos pessoais de proteção que, segundo Lisboa (2001), têm como função auxiliá-las diante dos riscos que possam dificultar o seu desenvolvimento. Assim, o autoconceito pode não estar funcionando como uma proteção para essas crianças e, ao mesmo tempo, pode estar interferindo na construção da auto-estima, pois, conforme as afirmações de Campbell e Lavallee (1993), e Harter (1993), os dois construtos são correlacionados positivamente com relação causal recíproca e sistemática.

Pereira (2001) aponta o autoconceito e a auto-estima positivos como elementos essenciais para o desenvolvimento da resiliência, como se afirmou anteriormente. Assim, as estratégias de coping empregadas estão diretamente ligadas ao autoconceito. Portanto, se a criança não conta com os recursos protetores pessoais, diante de uma situação estressora, pode estar ativando mecanismos ineficazes de coping.

As crianças vítimas de violência doméstica apresentaram uma avaliação mais positiva de si no que se refere a algumas questões relacionadas à aparência. Julgam-se fortes, populares e líderes, itens que pertencem à categoria aparência física e atributos, próprias de uma sociabilidade mais valorizada. Um dado semelhante foi observado por Stevenato et al. (2003), em estudo sobre autoconceito em crianças com dificuldades de aprendizagem e problemas de comportamento. Ambos os estudos envolveram crianças com problemas de comportamento. Segundo as referidas autoras, a valorização da aparência e atributos pode ser uma forma de compensação dos prejuízos reconhecidos quanto ao autoconceito.

A popularidade e a liderança relacionadas ao comportamento agressivo e anti-social foram referidas por Rodkin et al. (2000) como uma forma de participação social com valorização própria em grupos específicos. No presente estudo, podese levantar a hipótese de associação entre popularidade e dificuldades comportamentais, como agitação, agressividade e desobediência. Segundo os autores, o comportamento agressivo pode ser valorizado socialmente, dependendo do contexto. Se o comportamento com características anti-sociais está contribuindo para a popularidade das crianças vítimas de violência doméstica, elas podem estar correndo o risco de manutenção do comportamento agressivo e anti-social, devido à percepção de serem bem aceitas socialmente.

Por outro lado, é possível pensar que a avaliação positiva do autoconceito em aspectos relativos à liderança e à popularidade pode refletir recursos pessoais das crianças no enfrentamento dos estressores e das adversidades, possivelmente influenciando de forma positiva a maneira como se colocam frente às situações de seu cotidiano.

Em geral, as crianças sem história de risco psicossocial relatada, comparativamente às crianças vítimas de violência doméstica, fizeram uma avaliação mais positiva de si. Esse dado sugere que as crianças sem história de risco psicossocial relatada apresentaram mais recursos pessoais ligados à imagem que fazem de si, e isso pode favorecê-las ao lidarem com situações estressoras.

$\mathrm{Na}$ avaliação sistemática do desempenho escolar, os grupos não apresentaram diferenças quanto ao escore total. É importante destacar que a associação entre exposição à violência interparental denunciada e o desempenho acadêmico em crianças foi estudada por Brancalhone et al. (2004), e Kernic et al. (2002). Nestes dois estudos, não foram encontradas dificuldades com relação ao desempenho acadêmico. Os dados do presente estudo são concordantes com esses achados prévios.

Vale destacar, contudo, que a única diferença identificada quanto ao desempenho escolar foi na área da escrita, em que as crianças vítimas de violência doméstica apresentaram mais dificuldades. Considerando que o aprendizado da escrita depende, dentre outros fatores, da mediação social para ocorrer e que as crianças avaliadas apresentam recursos intelectuais preservados, conforme observado através de avaliação pelo Teste das Matrizes Progressivas de Raven, questiona-se até que ponto as dificuldades observadas não poderiam ser minimizadas com o suporte escolar adequado.

\section{Considerações finais}

$\mathrm{Na}$ idade escolar, as dificuldades de desempenho se configuram como condições de vulnerabilidade ao desenvolvimento, que requerem a ativação de recursos de proteção no sentido de minimizar o impacto negativo de tais dificuldades. Entre as crianças vítimas de violência doméstica, verificou-se que existem áreas do autoconceito em que não aparecem prejuízos em relação ao grupo de comparação, são elas: a aparência, a ansiedade, a popularidade e a felicidade. Também em relação ao desempenho escolar, observaram-se dificuldades apenas na área da escrita. Essas características sugerem que as crianças contam com recursos de proteção em determinadas áreas, contudo, mesmo apresentando recursos quanto ao autoconceito, ao desempenho escolar e ao nível intelectual, as crianças manifestam sinais de comprometimento em áreas essenciais do autoconceito, comportamento e status intelectual. Tais áreas relacionam-se à socialização e ao desempenho escolar, que constituem tarefas desenvolvimentais próprias deste período de vida. Portanto, as crianças mostram prejuízos em áreas que deveriam funcionar como proteção, configurando uma condição de vulnerabilidade, ou seja, não contam com alguns dos recursos essenciais para enfrentarem as tarefas desenvolvimentais da 
idade escolar.

É importante destacar os recursos identificados entre as crianças vítimas de violência doméstica que podem ser empregados no enfrentamento dos estressores e das adversidades de seu cotidiano, o que sugere a capacidade da criança de alcançar bons resultados, apesar de sérias ameaças ao seu desenvolvimento. Considera-se que isso se dá em função de fatores de proteção possivelmente associados aos recursos pessoais das crianças. Tal dado sugere, também, a possibilidade de essas crianças estarem suscetíveis a influências positivas, que podem ser proporcionadas por práticas de saúde mental focalizadas na criança, como o tratamento psicológico, bem como por meio de programas de prevenção com enfoque psicopedagógico que visem favorecer o desenvolvimento sadio das crianças.

Quanto à escola, tal como essa instituição se apresenta no momento, pode-se questionar se ela está suficientemente preparada para exercer seu papel de proteção, pois o desempenho escolar parece pouco aproveitado como variável do desenvolvimento. A presença de indicadores de autoconceito positivo em diversas áreas, mas com dificuldades nas áreas comportamento e status intelectual e acadêmico, mostra que é preciso considerar as autopercepcões das crianças como recurso para o enfrentamento das demandas da realidade, o que pode proporcionar mais confiança em si mesmas. Tal dado sugere a necessidade de se estimular práticas na escola que favoreçam o desenvolvimento do autoconceito como medida de saúde mental. No contexto escolar, o planejamento de programas de prevenção que valorizem as relações estabelecidas entre professores e alunos, o reconhecimento dos esforços acadêmicos e das potencialidades da criança, bem como o encorajamento para a sua integração social, representam importantes contribuições que a escola pode oferecer para a formação e modulação do autoconceito das crianças.

Analisando alguns dos cuidados metodológicos adotados, merecem considerações específicas os controles adotados na composição dos grupos quanto ao nível intelectual da criança, média de idade, escolaridade da criança e dos pais e nível socioeconômico da família. Isso possibilitou maior segurança na discussão dos dados, principalmente, no que se refere às condições socioeconômicas, frequentemente identificadas como associadas à classe social baixa.

A metodologia adotada no presente estudo apresentou algumas limitações. A primeira, quanto ao delineamento de corte transversal, pois não permitiu uma compreensão aprofundada sobre como as crianças vítimas de violência doméstica se desenvolveram. Tais informações só seriam possíveis por meio de um estudo longitudinal que pudesse fornecer, através de dados sobre mudanças e diferenças quanto ao desenvolvimento individual da criança vítima de violência, pistas sobre relações entre experiências anteriores de adversidades e suas conseqüências. A realização de estudos longitudinais permitirá dimensionar melhor os riscos a que estão expostas tais crianças ao longo do desenvolvimento, considerando o tempo decorrido após a violência, objetivando analisar o tempo que a criança e a família demoram a se recuperar e os processos de superação das adversidades, de modo a integrar a avaliação de mecanismos de risco e de proteção ao desenvolvimento infantil.

As crianças incluídas neste estudo constituem uma amostra procedente de uma população identificada, o que tem como implicação a maior tendência de que as notificações de violência registradas em órgãos de proteção sejam mais graves do que as ocorrências em amostras da comunidade. Isso, de certo modo, limita a generalização dos dados. Esse fato indica a importância de se estudar amostras da comunidade. Outra limitação consiste no fato de que a pesquisa foi realizada com uma amostra localizada, três anos após o atendimento, favorecendo uma perda amostral razoável que contribuiu para o seu tamanho reduzido.

Salienta-se que, no delineamento, priorizou-se a caracterização de variáveis pessoais das crianças, sem considerar as questões que envolvem as famílias, como a saúde mental dos pais e os recursos do ambiente familiar. Contudo, constata-se que tais variáveis, dependendo do contexto de ocorrência da violência doméstica, podem trazer outras implicações. Também não se exploraram os processos, ou seja, as interações entre indivíduo e ambiente, como as relações familiares, o enfrentamento por parte das famílias e das crianças, a percepção que a criança tem da situação e do suporte familiar e sua interpretação do evento estressor. Considera-se que são necessários outros estudos que busquem responder às questões levantadas, por meio de recursos metodológicos diversos, que enfoquem, também, amostras da comunidade, buscando analisar o desenvolvimento das crianças expostas à violência em outros contextos, como a escola e a comunidade.

\section{Referências}

Angelini, A. L., Alves, I. C. B., Custódio, E. M., Duarte, W. F., \& Duarte, K. L. M. (1999). Manual matrizes progressivas coloridas de Raven: escala especial. São Paulo: Centro Editor de Testes e Pesquisas em Psicologia.

Brancalhone, P. G., Fogo, J. C., \& Williams, L. C. A. (2004). Crianças expostas à violência conjugal: avaliação do desempenho acadêmico. Psicologia: Teoria e Pesquisa, 20(2), 113-117.

Campbell, J. D., \& Lavallee, L. F. (1993). Who am I? The role of self-concept confusion in understanding the behavior of people with low self-esteem. In R. F. Baumeister (Org.), Self-esteem: the puzzle of low self-regard (pp. 3-20). New York: Plenum.

Candieux, A. (1996). Psychometric properties of a Pictorial Self-Concept Scale among young learning disabled pupils. Psychology in the Schools, 33(3), 221-229.

Chapman, J. W. (1988). Learning disabled children's self-concepts. Review of Educational Research, 58, 347-371.

Cicchetti, D., \& Rizley, R. (1981). Developmental perspectives on the etiology, intergenerational transmission, and sequelae of child maltreatment. New Directions for Child Development, 11, 32-59.

Deslandes, S. F. (1994). Prevenir a violência: um desafio para profissionais de saúde. Rio de Janeiro: FIOCRUZ.

Erikson, E. (1976). Infância e sociedade. Tradução G. Amado. Rio de Janeiro: Zahar.

Gresham, F. M., Lane, K. L., Mac Millan, D. L., Bocian, K. M., \& Ward, S. L. (2000). Effects of positive and negative illusory biases: comparisons across social and academic self-concept domains. Journal of School Psychology, $38(2), 151-175$.

Harter, S. (1993). Causes and consequences of low self-esteem in children and 
adolescents. In R. F. Baumeister (Org.), Self-esteem: the puzzle of low selfregard (pp.87-116). New York: Plenum.

Jacob, A. V. (2001). O desempenho escolar e suas relações com autoconceito e auto-eficácia. Tese de Doutorado, Faculdade de Medicina de Ribeirão Preto, Universidade de São Paulo, Ribeirão Preto.

Jacob, A. V., \& Loureiro, S. R. (1996). Desenvolvimento Afetivo: o processo de aprendizagem e o atraso escolar. Paidéia: Cadernos de Psicologia e Educação, 10/11, 149-160.

Jacob, A. V., \& Loureiro, S. R. (1999). Autoconceito e o desempenho escolar [Resumo]. Sociedade Brasileira de Psicologia (Org.), XXIX Reunião Anual de Psicologia. Resumos (p. 164). Campinas: Universidade de São Paulo.

Kemic, M.A. Holt, V.L., Wolf, M.E.., McKnight, B., Huebner, C. E., \& Rivara, F.P. (2002). Academic and school health issues among children exposed to maternal intimate partner abuse. Archives of Pediatrics and Adolescent Medicine, 156(6), 549-555

Lisboa, C. S. M. (2001). Estratégias de coping e agressividade: um estudo comparativo entre crianças vítimas e não vítimas de violência doméstica. Dissertação de Mestrado, Universidade Federal do Rio Grande do Sul, Porto Alegre.

Masten, A. S. (2001). Ordinary magic resilience processes in development. American Psychologist, 56(3), 227-238.

McCloskey, L. A., Figueiredo, A. J., \& Koss, M. P. (1995). The effects of systemic family violence on children's mental health. Child Development, 66(5), 1239-1261.

Milani, R. G., \& Loureiro, S. R. (2008). Famílias e violência doméstica: condições psicossociais pós ações do Conselho Tutelar. Psicologia Ciência e Profissão, 28(1), 50-67.

Oliveira, G. C. (2000). Autoconceito do adolescente. In F. F. Sisto, G. C. Oliveira,
\& L. D. T. Fini (Orgs.), Leituras de psicologia para formação de professores (pp. 58-69). Petrópolis: Vozes.

Pereira, A. M. S. (2001). Resiliência, personalidade, stress e estratégias de coping. In J. Tavares (Org.), Resiliência e educação (pp. 77-94). São Paulo: Cortez.

Piers, E. V. (1984). Piers-Harris Children's Self-Concept Scale-revised manual. Los Angeles: Counselor Psychological Tests/WPS

Rodkin, P. C., Farmer, T. W., Pearl, R., \& Arcker, R. V. (2000). Heterogeneity of popular boys: antisocial and prosocial configurations. Developmental Psychology, 36(1), 14-24.

Roque, E. M. S. T., \& Ferriani, M. G. C. (2002). Desvendando a violência doméstica contra crianças e adolescentes sob a ótica dos operadores do direito na comarca de Jardinópolis-SP. Revista Latino-Americana de Enfermagem, 10(3), 334-344

Salzinger, S., Feldman, R. S., Ng-Mak, D. S., Mojica, E., \& Stockhammer, T. F. (2001). The effect of physical abuse on children's social and affective status: a model of cognitive and behavioral processes explaining the association. Development \& Psychopathology, 13(4), 805-825.

Shonk, S.M., \& Cicchetti, D. (2001). Maltreatment, competency deficits, and risk for academic and behavioral maladjustment. Development \& Psychopathology, 37(1), 3-17.

Stein, L. M. (1994). TDE: teste de desempenho escolar. São Paulo: Casa do Psicólogo.

Stevenato, I. S., Loureiro, S. L., Linhares, M. B. M., \& Marturano, E. M. (2003). Autoconceito de crianças com dificuldades de aprendizagem e problemas de comportamento. Psicologia em Estudo, 8(1), 67-76.

Szyndrowski, D. (1999). The impact of domestic violence on adolescent aggression in the schools. Preventing School Failure, 44(1), 9-14

Rute Grossi Milani, doutora em Saúde Mental pela Faculdade de Medicina de Ribeirão Preto (FMRP - USP), é psicóloga e professora do Centro Universitário de Maringá. Endereço para correspondência: Clínica de Psicologia do Centro Universitário de Maringá, Av. Guedner, 1610, Jd. Aclimação, Maringá-PR. CEP: 87.050-390. Fone/Fax: (44) 3027-6360 ramal 183

E-mail: rute@cesumar.br

Sonia Regina Loureiro, doutora em Psicologia Clínica pelo Instituto de Psicologia da Universidade de São Paulo (USP), é professora do Departamento de Neurociências e Ciências do Comportamento, da Faculdade de Medicina de Ribeirão Preto (FMRP-USP). E-mail: srlourei@fmrp.usp.br 